\title{
A positive combinatorial formula for the complexity of the $q$-analog of the $n$-cube
}

\author{
Murali K. Srinivasan \\ Department of Mathematics \\ Indian Institute of Technology, Bombay \\ Powai, Mumbai 400076, INDIA \\ mks@math.iitb.ac.in \\ murali.k.srinivasan@gmail.com
}

Submitted: Feb 6, 2012; Accepted: May 25, 2012; Published: Jun 6, 2012

Mathematics Subject Classifications: 05E25, 05C50

To the memory of Mini

\begin{abstract}
The number of spanning trees of a graph $G$ is called the complexity of $G$ and denoted $c(G)$. A classical result in algebraic graph theory explicitly diagonalizes the Laplacian of the $n$-cube $C(n)$ and yields, using the Matrix-Tree theorem, an explicit formula for $c(C(n))$. In this paper we explicitly block diagonalize the Laplacian of the $q$-analog $C_{q}(n)$ of $C(n)$ and use this, along with the Matrix-Tree theorem, to give a positive combinatorial formula for $c\left(C_{q}(n)\right)$. We also explain how setting $q=1$ in the formula for $c\left(C_{q}(n)\right)$ recovers the formula for $c(C(n))$.
\end{abstract}

\section{Introduction}

The number of spanning trees of a graph $G$ is called the complexity of $G$ and denoted $c(G)$. The hypercube $C(n)$ is the graph whose vertex set is the set $B(n)$ of all subsets of the $n$-set $\{1,2, \ldots, n\}$ and where two subsets $X, Y \in B(n)$ are connected by an edge iff $X \subseteq Y$ or $Y \subseteq X$, and $\| X|-| Y||=1$. A classical result in algebraic graph theory (see Example 5.6.10 in [St2]) states that

$$
c(C(n))=\frac{1}{2^{n}}\left\{\prod_{k=1}^{n}(2 k)^{\left(\begin{array}{l}
n \\
k
\end{array}\right)}\right\}=\prod_{k=2}^{n}(2 k)^{\left(\begin{array}{l}
n \\
k
\end{array}\right)} .
$$


We now define a $q$-analog of $C(n)$. Let $q$ be a prime power and let $B_{q}(n)$ denote the set of all subspaces of an $n$-dimensional vector space over the finite field $\mathbb{F}_{q}$. Define $[k]_{q}=1+q+q^{2}+\cdots+q^{k-1}, k \in \mathbb{N}=\{0,1,2, \ldots\}$. For $0 \leqslant k \leqslant n$, the $q$-binomial coefficient

$$
\left[\begin{array}{c}
n \\
k
\end{array}\right]_{q}=\frac{[1]_{q}[2]_{q} \cdots[n]_{q}}{[1]_{q} \cdots[k]_{q}[1]_{q} \cdots[n-k]_{q}}
$$

denotes the number of $k$-dimensional subspaces in $B_{q}(n)$. The q-analog $C_{q}(n)$ of the hypercube is the graph whose vertex set is $B_{q}(n)$ and where subspaces $X, Y \in B_{q}(n)$ are connected by an edge iff $X \subseteq Y$ or $Y \subseteq X$, and $|\operatorname{dim}(X)-\operatorname{dim}(Y)|=1$. The number of vertices of $C_{q}(n)$ is the Galois number

$$
G_{q}(n)=\sum_{k=0}^{n}\left[\begin{array}{l}
n \\
k
\end{array}\right]_{q} .
$$

In this paper we present a positive, combinatorial formula for $c\left(C_{q}(n)\right)$. While this formula is not as explicit as (1) it can be used to efficiently write down $c\left(C_{q}(n)\right)$ for a given $n$.

Let us first reformulate the original formula (1) for $c(C(n))$ in order to bring out the similarity with our formula for $c\left(C_{q}(n)\right)$. We have

$$
\begin{aligned}
c(C(n)) & =\frac{1}{2^{n}}\left\{\prod_{k=1}^{n}(2 k)^{\left.\left(\begin{array}{l}
n \\
k
\end{array}\right)\right\}}\right. \\
& =\frac{1}{2^{n}}\left\{\prod_{k=1}^{n}(2 k)\right\}\left\{\prod_{k=1}^{\lfloor n / 2\rfloor}\left(\prod_{j=k}^{n-k}(2 j)\right)^{\left(\begin{array}{l}
n \\
k
\end{array}\right)-\left(\begin{array}{c}
n \\
k-1
\end{array}\right)}\right\} \\
& =n !\left\{\prod_{k=1}^{\lfloor n / 2\rfloor}\left(\prod_{j=k}^{n-k}(2 j)\right)^{\left(\begin{array}{l}
n \\
k
\end{array}\right)-\left(\begin{array}{c}
n \\
k-1
\end{array}\right)}\right\} .
\end{aligned}
$$

To see the equivalence of the first and second lines above note that, for $1 \leqslant j \leqslant n / 2$, the exponent of $2 j$ in the base of the first line is $\left(\begin{array}{l}n \\ j\end{array}\right)$ and in the base of the second line is also $\left(\begin{array}{l}n \\ j\end{array}\right)=1+\left(\begin{array}{l}n \\ 1\end{array}\right)-\left(\begin{array}{l}n \\ 0\end{array}\right)+\cdots+\left(\begin{array}{c}n \\ j\end{array}\right)-\left(\begin{array}{c}n \\ j-1\end{array}\right)$. Since $\left(\begin{array}{l}n \\ k\end{array}\right)=\left(\begin{array}{c}n \\ n-k\end{array}\right)$ the same conclusion holds for $n / 2 \leqslant j \leqslant n$.

Let $k, n \in \mathbb{N}$ with $k \leqslant n / 2$. For $k \leqslant j \leqslant n-k+1$, define polynomials $F_{q}(n, k, j)$ in $q$ with integral coefficients using the following recursion:

$$
\begin{aligned}
F_{q}(n, k, n-k+1) & =1, \\
F_{q}(n, k, n-k) & =[k]_{q}+[n-k]_{q},
\end{aligned}
$$

and, for $k \leqslant j<n-k$,

$$
\begin{aligned}
& F_{q}(n, k, j)= \\
& \quad\left([j]_{q}+[n-j]_{q}\right) F_{q}(n, k, j+1)-\left(q^{k}[j+1-k]_{q}[n-k-j]_{q}\right) F_{q}(n, k, j+2) .
\end{aligned}
$$


In Section 3 we prove the following formula for $c\left(C_{q}(n)\right)$. It is similar to formula (2) above except that the explicit term $\prod_{j=k}^{n-k}(2 j)$ is replaced by a recursive calculation.

Theorem 1.1 We have

$$
c\left(C_{q}(n)\right)=[1]_{q}[2]_{q} \cdots[n]_{q}\left\{\prod_{k=1}^{\lfloor n / 2\rfloor} F_{q}(n, k, k)^{\left[\begin{array}{l}
n \\
k
\end{array}\right]_{q}-\left[\begin{array}{c}
n \\
k-1
\end{array}\right]_{q}}\right\} .
$$

The following table, computed using Maple, gives the first five values of $c\left(C_{q}(n)\right)$.

$$
\begin{aligned}
c\left(C_{q}(1)\right)= & 1, \\
c\left(C_{q}(2)\right)= & {[2]_{q} 2^{q} } \\
c\left(C_{q}(3)\right)= & {[2]_{q}[3]_{q}\left(4+3 q+q^{2}\right)^{q(1+q)}, } \\
c\left(C_{q}(4)\right)= & {[2]_{q}[3]_{q}[4]_{q}\left(8+12 q+12 q^{2}+10 q^{3}+4 q^{4}+2 q^{5}\right)^{q\left(1+q+q^{2}\right)} } \\
& \times(2+2 q)^{q^{2}\left(q^{2}+1\right)}, \\
c\left(C_{q}(5)\right)= & {[2]_{q}[3]_{q}[4]_{q}[5]_{q} F_{q}(5,1,1)^{q(1+q)\left(1+q^{2}\right)} } \\
& \times F_{q}(5,2,2)^{q^{2}\left(1+q+q^{2}+q^{3}+q^{4}\right)},
\end{aligned}
$$

where $F_{q}(5,2,2)=4+8 q+7 q^{2}+4 q^{3}+q^{4}$ and

$$
F_{q}(5,1,1)=16+36 q+53 q^{2}+65 q^{3}+69 q^{4}+58 q^{5}+42 q^{6}+26 q^{7}+13 q^{8}+5 q^{9}+q^{10} .
$$

The table above suggests that the formula for $c\left(C_{q}(n)\right)$ in Theorem 1.1 is positive, i.e., for $k \leqslant n / 2$, both $\left[\begin{array}{c}n \\ k\end{array}\right]_{q}-\left[\begin{array}{c}n \\ k-1\end{array}\right]_{q}$ and $F_{q}(n, k, k)$ have nonnegative coefficients. A special case of a result of Butler $[\mathbf{B}]$ shows that indeed $\left[\begin{array}{l}n \\ k\end{array}\right]_{q}-\left[\begin{array}{c}n \\ k-1\end{array}\right]_{q}, k \leqslant n / 2$ has nonnegative coefficients.

In Section 4 we give an explicit combinatorial formula for $F_{q}(n, k, j)$ and prove the following result.

Theorem $1.2 F_{q}(n, k, j)$ has nonnegative coefficients for $0 \leqslant k \leqslant n / 2, k \leqslant j \leqslant n-k+1$.

We add here a remark on our notation which may be nonstandard in the algebraic graph theory literature. The notations $C(n)$ and $B(n)$ are from [St2, St1], except that they are called $C_{n}$ and $B_{n}$ there. This explains our notations $C_{q}(n)$ and $B_{q}(n)$ for the $q$-analogs. The Grassmann graphs (also called $q$-Johnson graphs) $C_{q}(n, m)$ in Section 4 are normally denoted $J_{q}(n, m)$ (or by some variant) whereas in this paper the symbol $J$ stands for a Jordan basis.

In the next section we discuss the framework of orthogonal symmetric Jordan bases with respect to the up operator on a graded poset. 


\section{Singular values}

In this section we recall a result of Terwilliger $[\mathbf{T}]$ and give a proof of it based on Proctor's $\mathfrak{s l}(2, \mathbb{C})$ technique $[\mathbf{P}]$.

Let $P$ be a finite graded poset with rank function $r: P \rightarrow \mathbb{N}$ (our poset terminology follows [St1]). The rank of $P$ is $r(P)=\max \{r(p): p \in P\}$ and, for $i=0,1, \ldots, r(P), P_{i}$ denotes the set of elements of $P$ of rank $i$.

For a finite set $S$, let $V(S)$ denote the complex vector space with $S$ as basis. Let $P$ be a graded poset with $n=r(P)$. Then we have $V(P)=V\left(P_{0}\right) \oplus V\left(P_{1}\right) \oplus \cdots \oplus V\left(P_{n}\right)$ (vector space direct sum). An element $v \in V(P)$ is homogeneous if $v \in V\left(P_{i}\right)$ for some $i$, and if $v \neq 0$, we extend the notion of rank to nonzero homogeneous elements by writing $r(v)=i$. The up operator $U: V(P) \rightarrow V(P)$ is defined, for $p \in P$, by $U(p)=\sum_{p^{\prime}} p^{\prime}$, where the sum is over all $p^{\prime}$ covering $p$. A symmetric Jordan chain (SJC) in $V(P)$ is a sequence

$$
s=\left(v_{k}, \ldots, v_{n-k}\right), \quad k \leqslant n / 2,
$$

of nonzero homogeneous elements of $V(P)$ such that $r\left(v_{i}\right)=i$ for $i=k, \ldots, n-k$, $U\left(v_{i-1}\right)=v_{i}$, for $i=k+1, \ldots, n-k$, and $U\left(v_{n-k}\right)=0$ (note that the elements of this sequence are linearly independent, being nonzero and of different ranks). We say that $s$ starts at rank $k$ and ends at rank $n-k$. A symmetric Jordan basis (SJB) of $V(P)$ is a basis of $V(P)$ consisting of a disjoint union of symmetric Jordan chains in $V(P)$.

Let $\langle$,$\rangle denote the standard inner product on V(P)$, i.e., $\left\langle p, p^{\prime}\right\rangle=\delta\left(p, p^{\prime}\right)$ (Kronecker delta) for $p, p^{\prime} \in P$. The length $\sqrt{\langle v, v\rangle}$ of $v \in V(P)$ is denoted $\|v\|$.

Suppose we have an orthogonal SJB $J(n)$ of $V(P)$. Normalize the vectors in $J(n)$ to get an orthonormal basis $J^{\prime}(n)$. Let $\left(v_{k}, \ldots, v_{n-k}\right)$ be a SJC in $J(n)$. Put $v_{u}^{\prime}=\frac{v_{u}}{\left\|v_{u}\right\|}$ and

$\alpha_{u}=\frac{\left\|v_{u+1}\right\|}{\left\|v_{u}\right\|}, k \leqslant u \leqslant n-k$ (we set $v_{k-1}^{\prime}=v_{n-k+1}=0$ ). We have, for $k \leqslant u \leqslant n-k$,

$$
U\left(v_{u}^{\prime}\right)=\frac{U\left(v_{u}\right)}{\left\|v_{u}\right\|}=\frac{v_{u+1}}{\left\|v_{u}\right\|}=\alpha_{u} v_{u+1}^{\prime}
$$

Thus the matrix of $U$ with respect to $J^{\prime}(n)$ is in block diagonal form, with a block corresponding to each (normalized) SJC in $J(n)$, and with the block corresponding to $\left(v_{k}^{\prime}, \ldots, v_{n-k}^{\prime}\right)$ above being a lower triangular matrix with subdiagonal $\left(\alpha_{k}, \ldots, \alpha_{n-k-1}\right)$ and 0's elsewhere.

The down operator $D: V(P) \rightarrow V(P)$ is defined, for $p \in P$, by $D(p)=\sum_{p^{\prime}} p^{\prime}$, where the sum is over all $p^{\prime}$ covered by $p$. Note that the matrices, with respect to the standard basis $P$, of $U$ and $D$ are real and transposes of each other. Since $J^{\prime}(n)$ is orthonormal with respect to the standard inner product, it follows that the matrices of $U$ and $D$, with respect to the basis $J^{\prime}(n)$, must be adjoints of each other. Thus the matrix of $D$ with respect to $J^{\prime}(n)$ is in block diagonal form, with a block corresponding to each (normalized) SJC in $J(n)$, and with the block corresponding to $\left(v_{k}^{\prime}, \ldots, v_{n-k}^{\prime}\right)$ above being 
an upper triangular matrix with superdiagonal $\left(\alpha_{k}, \ldots, \alpha_{n-k-1}\right)$ and 0's elsewhere. So, for $k-1 \leqslant u \leqslant n-k-1$, we have

$$
D\left(v_{u+1}^{\prime}\right)=\alpha_{u} v_{u}^{\prime}
$$

In particular, the subspace spanned by $\left\{v_{k}, \ldots, v_{n-k}\right\}$ is closed under $U$ and $D$.

Another useful observation is the following: take scalars $\beta_{0}, \beta_{1}, \ldots, \beta_{n}$ and define the operator $\gamma: V(P) \rightarrow V(P)$ by $\gamma(p)=\beta_{r(p)} p, p \in P$. Since each element of the SJC $\left(v_{k}, \ldots, v_{n-k}\right)$ is homogeneous, it follows from the definition of $\gamma$ that the subspace spanned by $\left\{v_{k}, \ldots, v_{n-k}\right\}$ is closed under $U, D$ and $\gamma$.

The Boolean algebra is the graded poset of rank $n$ obtained by partially ordering $B(n)$ by containment (with rank of a subset given by cardinality). The q-analog of the Boolean algebra is obtained by partially ordering $B_{q}(n)$ by inclusion. This gives a graded poset of rank $n$ with rank of a subspace given by dimension.

Theorem 2.1 (Terwilliger [T], Item 5 of Theorem 3.3)

There exists a $S J B J(q, n)$ of $V\left(B_{q}(n)\right)$ such that

(i) The elements of $J(q, n)$ are orthogonal with respect to $\langle$,$\rangle (the standard inner product).$

(ii) (Singular Values) Let $0 \leqslant k \leqslant n / 2$ and let $\left(v_{k}, \ldots, v_{n-k}\right)$ be any SJC in $J(q, n)$ starting at rank $k$ and ending at rank $n-k$. Then we have, for $k \leqslant u<n-k$,

$$
\frac{\left\|v_{u+1}\right\|}{\left\|v_{u}\right\|}=\sqrt{q^{k}[u+1-k]_{q}[n-k-u]_{q}} .
$$

Proof The existence of an orthogonal SJB follows from standard well known results (recalled in parts (a) and (b) below). To determine the singular values we use the $\mathfrak{s l}(2, \mathbb{C})$ technique of Proctor $[\mathbf{P}]$. The expression for the singular values written down in $[\mathbf{T}]$ (see item 5 on top of page 208) can be easily shown to equal the one given here.

(a) The map $U^{n-2 k}: V\left(B_{q}(n)_{k}\right) \rightarrow V\left(B_{q}(n)_{n-k}\right), 0 \leqslant k \leqslant n / 2$ is well known to be bijective (see $[\mathbf{K}])$. It follows, using a standard Jordan canonical form argument (see Chapter 6 of $[\mathbf{E}])$, that an SJB of $V\left(B_{q}(n)\right)$ exists.

(b) Now we show existence of an orthogonal SJB. We use the action of the group $G L\left(n, \mathbb{F}_{q}\right)$ on $B_{q}(n)$. As is easily seen the existence of an orthogonal SJB of $V\left(B_{q}(n)\right)$ (under the standard inner product) follows from facts (i)-(iv) below by an application of Schur's lemma:

(i) Existence of some SJB of $V\left(B_{q}(n)\right)$.

(ii) $U$ is $G L\left(n, \mathbb{F}_{q}\right)$-linear.

(iii) For $0 \leqslant k \leqslant n, V\left(B_{q}(n)_{k}\right)$ is a multiplicity free $G L\left(n, \mathbb{F}_{q}\right)$-module (this is well known. The corresponding result for the symmetric group action on $V\left(B(n)_{k}\right)$ is proved in Chapter 29 of [JL]. An identical proof works here).

(iv) For a finite group $G$, a $G$-invariant inner product on an irreducible $G$-module is unique up to scalars. 
(c) Now we prove part (ii) of the Theorem. Define an operator $H: V\left(B_{q}(n)\right) \rightarrow$ $V\left(B_{q}(n)\right)$ by

$$
H(X)=\left([k]_{q}-[n-k]_{q}\right) X, \quad X \in B_{q}(n)_{k}, 0 \leqslant k \leqslant n .
$$

It is easy to check that $[U, D]=U D-D U=H$. To see this, fix $X \in B_{q}(n)_{k}$, and note that $U D(X)=[k]_{q} X+\sum_{Y} Y$, where the sum is over all $Y \in B_{q}(n)_{k}$ with $\operatorname{dim}(X \cap Y)=k-1$. Similarly, $D U(X)=[n-k]_{q} X+\sum_{Y} Y$, where the sum is over all $Y \in B_{q}(n)_{k}$ with $\operatorname{dim}(X \cap Y)=k-1$. Subtracting we get $[U, D]=H$.

Let $J(q, n)$ be an orthogonal SJB of $V\left(B_{q}(n)\right)$ and let $\left(v_{k}, \ldots, v_{n-k}\right)$ be a SJC in $J(q, n)$ starting at rank $k$ and ending at rank $n-k$. Put $v_{j}^{\prime}=\frac{v_{j}}{\left\|v_{j}\right\|}$ and $\alpha_{j}=\frac{\left\|v_{j+1}\right\|}{\left\|v_{j}\right\|}, k \leqslant j \leqslant n-k$. We have, from (7) and (8),

$$
U\left(v_{j}^{\prime}\right)=\alpha_{j} v_{j+1}^{\prime}, \quad D\left(v_{j+1}^{\prime}\right)=\alpha_{j} v_{j}^{\prime}, \quad k \leqslant j<n-k .
$$

We need to show that

$$
\alpha_{j}^{2}=q^{k}[j+1-k]_{q}[n-k-j]_{q}, \quad k \leqslant j<n-k .
$$

We show this by induction on $j$. We have $D U=U D-H$. Now $D U\left(v_{k}^{\prime}\right)=\alpha_{k} D\left(v_{k+1}^{\prime}\right)=$ $\alpha_{k}^{2} v_{k}^{\prime}$ and $(U D-H)\left(v_{k}^{\prime}\right)=\left([n-k]_{q}-[k]_{q}\right) v_{k}^{\prime}\left(\right.$ since $\left.D\left(v_{k}^{\prime}\right)=0\right)$. Hence $\alpha_{k}^{2}=[n-k]_{q}-$ $[k]_{q}=q^{k}[n-2 k]_{q}$. Thus (10) holds for $j=k$.

As in the previous paragraph $D U\left(v_{j}^{\prime}\right)=\alpha_{j}^{2} v_{j}^{\prime}$ and $(U D-H)\left(v_{j}^{\prime}\right)=\left(\alpha_{j-1}^{2}+[n-j]_{q}-\right.$ $\left.[j]_{q}\right) v_{j}^{\prime}$. By induction, we may assume $\alpha_{j-1}^{2}=q^{k}[j-k]_{q}[n-k-j+1]_{q}$. Thus we see that

$$
\begin{aligned}
\alpha_{j}^{2}= & q^{k}[j-k]_{q}[n-k-j+1]_{q}+[n-j]_{q}-[j]_{q} \\
= & q^{k}\left\{\left([j+1-k]_{q}-q^{j-k}\right)\left([n-k-j]_{q}+q^{n-k-j}\right)\right\}+[n-j]_{q}-[j]_{q} \\
= & q^{k}\left\{[j+1-k]_{q}[n-k-j]_{q}+q^{n-k-j}[j+1-k]_{q}-q^{j-k}[n-k-j]_{q}-q^{n-2 k}\right\} \\
& +[n-j]_{q}-[j]_{q} \\
= & q^{k}[j+1-k]_{q}[n-k-j]_{q}+q^{n-j}[j+1-k]_{q}-\left(q^{j}[n-k-j]_{q}+q^{n-k}\right) \\
& +[n-j]_{q}-[j]_{q} \\
= & q^{k}[j+1-k]_{q}[n-k-j]_{q}+[n+1-k]_{q}-[n-j]_{q}-\left([n+1-k]_{q}-[j]_{q}\right) \\
& +[n-j]_{q}-[j]_{q} \\
= & q^{k}[j+1-k]_{q}[n-k-j]_{q},
\end{aligned}
$$

completing the proof.

\section{Complexity of $C_{q}(n)$}

In this section we apply the block diagonalization technique of Schrijver $[\mathbf{S}]$ to prove Theorem 1.1. 
Before proceeding further we recall the matrix tree theorem (see Theorem 5.6.8 in [St2]). Let $G$ be a simple graph on the vertex set $S$. Define the degree and adjacency operators $\operatorname{deg}, A: V(S) \rightarrow V(S)$ of $G$ as follows : for $v \in S, \operatorname{deg}(v)=($ degree of $v) v$ and $A(v)=\sum u$, the sum being over all $u \in S$ adjacent to $v$ in $G$. The Laplacian operator $L$ of $G$ is defined by $L=\operatorname{deg}-A$. Assume $G$ is connected. It is known that 0 is an eigenvalue of $L$ of multiplicity 1 . The matrix tree theorem states that

$$
c(G)=\frac{\text { product of the nonzero eigenvalues of } L}{\# S}
$$

If $\bar{c}(G)$ denotes the number of rooted spanning trees (i.e., a spanning tree plus a choice of a vertex as a root) then the product of the nonzero eigenvalues of $L$ equals $\bar{c}(G)$.

The eigenvalues of the Laplacian of $C(n)$ are well known to be $2 k, k=0, \ldots, n$, with respective multiplicities $\left(\begin{array}{l}n \\ k\end{array}\right)$ (see Example 5.6.10 in [St2]), thereby explaining (1). The eigenvalues of the Laplacian of $C_{q}(n)$ are not known but we will give a formula for the product of the nonzero eigenvalues.

For $0 \leqslant k \leqslant n / 2$, define a real, symmetric, tridiagonal matrix $N_{q}(n, k)$ of size $n-2 k+1$, with rows and columns indexed by the set $\{k, k+1, \ldots, n-k\}$, and with entries given as follows: for $k \leqslant i, j \leqslant n-k$ define

$$
N_{q}(n, k)(i, j)= \begin{cases}-\sqrt{q^{k}[j-k]_{q}[n-k-j+1]_{q}} & \text { if } i=j-1, \\ {[j]_{q}+[n-j]_{q}} & \text { if } i=j \\ -\sqrt{q^{k}[j+1-k]_{q}[n-k-j]_{q}} & \text { if } i=j+1 \\ 0 & \text { if }|i-j| \geqslant 2 .\end{cases}
$$

Let $0 \leqslant k \leqslant n / 2$. For $k \leqslant j \leqslant n-k+1$ define $N_{q}(n, k, j)$ to be the principal submatrix of $N_{q}(n, k)$ indexed by the rows and columns in the set $\{j, j+1, \ldots, n-k\}$. Similarly, for $k-1 \leqslant j \leqslant n-k$ define $M_{q}(n, k, j)$ to be the principal submatrix of $N_{q}(n, k)$ indexed by the rows and columns in the set $\{k, j+1, \ldots, j\}$. Thus $N_{q}(n, k, k)=M_{q}(n, k, n-k)=$ $N_{q}(n, k)$ and $N_{q}(n, k, k+1), M_{q}(n, k, k-1)$ are empty matrices, which by convention have determinant 1 .

Before stating the next result we recall the following standard recursion for the determinants of tridiagonal matrices: Let $X=\left(x_{i j}\right)$ be a square tridiagonal matrix, $X^{\prime}$ be $X$ with first row, first column removed, and $X^{\prime \prime}$ be $X$ with first two rows, first two columns removed. Then $\operatorname{det}(X)=x_{11} \operatorname{det}\left(X^{\prime}\right)-x_{12} x_{21} \operatorname{det}\left(X^{\prime \prime}\right)$.

Lemma 3.1 We have

(i) $F_{q}(n, k, j)=\operatorname{det}\left(N_{q}(n, k, j)\right), 0 \leqslant k \leqslant n / 2, k \leqslant j \leqslant n-k+1$.

(ii) $F_{q}(n, 0, j)=[n]_{q}[n-1]_{q} \cdots[j]_{q}, 0 \leqslant j \leqslant n+1$.

(iii) $\operatorname{det}\left(M_{q}(n, 0, j)\right)=[n]_{q}[n-1]_{q} \cdots[n-j]_{q},-1 \leqslant j \leqslant n$. 
(iv) The sum of the determinants of all $n \times n$ principal submatrices of $N_{q}(n, 0)$ is equal to $\left([1]_{q}[2]_{q} \cdots[n]_{q}\right) G_{q}(n)$.

Proof (i) By (reverse) induction on $j$. The base cases $j=n-k+1, n-k$ are clear from (3), (4) and the general case follows by the recursion for the determinant of the tridiagonal matrix $N_{q}(n, k, j)$.

(ii) By (reverse) induction on $j$. The base cases $j=n+1, n$ are clear. By induction and the defining recurrence for $F_{q}(n, k, j)$ we have

$$
\begin{aligned}
F_{q}(n, 0, j) & =\left([j]_{q}+[n-j]_{q}\right) F_{q}(n, 0, j+1)-\left([j+1]_{q}[n-j]_{q}\right) F_{q}(n, 0, j+2) \\
& =\left([j]_{q}+[n-j]_{q}\right)[n]_{q} \cdots[j+1]_{q}-\left([j+1]_{q}[n-j]_{q}\right)[n]_{q} \cdots[j+2]_{q} \\
& =[n]_{q} \cdots[j]_{q} .
\end{aligned}
$$

(iii) Treat the diagonal entries of $N_{q}(n, 0)$ as a row vector of length $n+1$. Then this vector is symmetric, i.e., reads the same from left to right as right to left. Similarly for the subdiagonal and superdiagonal vectors. The result now follows from parts (i) and (ii).

(iv) For $0 \leqslant j \leqslant n$, deleting row and column $j$ from $N_{q}(n, 0)$ leaves a block diagonal matrix with blocks $N_{q}(n, 0, j+1)$ and $M_{q}(n, 0, j-1)$. Thus, the sum of the determinants of all $n \times n$ principal submatrices of $N_{q}(n, k)$ is equal to

$$
\begin{aligned}
\sum_{j=0}^{n} \operatorname{det}\left(N_{q}(n, 0, j+1)\right) \operatorname{det}\left(M_{q}(n, 0, j-1)\right) \\
=\sum_{j=0}^{n}[n]_{q}[n-1]_{q} \cdots[j+1]_{q}[n]_{q}[n-1]_{q} \cdots[n-j+1]_{q} \\
=\left([1]_{q}[2]_{q} \cdots[n]_{q}\right)\left\{\sum_{j=0}^{n}\left[\begin{array}{l}
n \\
j
\end{array}\right]_{q}\right\} \\
=\left([1]_{q}[2]_{q} \cdots[n]_{q}\right) G_{q}(n),
\end{aligned}
$$

completing the proof.

Proof (of Theorem 1.1): We can write the Laplacian operator $L$ of $C_{q}(n)$ as $L=$ deg$U-D$, where $U, D$ are the up and down operators on $V\left(B_{q}(n)\right)$.

Let $J(q, n)$ be a SJB of $V\left(B_{q}(n)\right)$ satisfying the conditions of Theorem 2.1. Normalize $J(q, n)$ to get an orthonormal basis $J^{\prime}(q, n)$. The degree of a vertex $X$ of $C_{q}(n)$ is $[k]_{q}+$ $[n-k]_{q}$, where $k=\operatorname{dim}(X)$. Since the vertex degrees are constant on $B_{q}(n)_{k}$ it follows that the subspace spanned by each SJC in $J(q, n)$ is closed under $L$. Using part (ii) of Theorem 2.1 we can write down the matrix of $L$ in the basis $J^{\prime}(q, n)$.

Let $0 \leqslant k \leqslant n / 2$. Let $\left(v_{k}, \ldots, v_{n-k}\right)$ be a (normalized) SJC in $J^{\prime}(q, n)$ starting at rank $k$. Let $W$ be the subspace spanned by $\left\{v_{k}, \ldots, v_{n-k}\right\}$. Then $W$ is invariant under $L$.

It follows from Theorem 2.1 that $N_{q}(n, k)$ is the matrix of $L: W \rightarrow W$ with respect to the (ordered) basis $\left\{v_{k}, \ldots, v_{n-k}\right\}$ (we take coordinate vectors with respect to a basis 
as column vectors). Thus the matrix of $L$ with respect to (a suitable ordering of) $J^{\prime}(q, n)$ is in block diagonal form, with blocks $N_{q}(n, k)$, for all $0 \leqslant k \leqslant n / 2$, and each such block is repeated $\left[\begin{array}{l}n \\ k\end{array}\right]_{q}-\left[\begin{array}{c}n \\ k-1\end{array}\right]_{q}$ times. The number of distinct blocks is $1+\lfloor n / 2\rfloor$.

It follows from parts (i), (ii) of Lemma 3.1 that $N_{q}(n, 0)$ is singular. Since $C_{q}(n)$ is connected we see that $N_{q}(n, k), 0<k \leqslant n / 2$ are all nonsingular and $N_{q}(n, 0)$ has 0 as an eigenvalue of multiplicity 1 . The result now follows from the matrix tree theorem and parts (i) and (iv) of Lemma 3.1.

Remark Since the subdiagonal entries of $N_{q}(n, k), 0 \leqslant k \leqslant n / 2$ are nonzero it easily follows that any eigenspace will have dimension 1 and thus $N_{q}(n, k)$ has $n-2 k+1$ distinct eigenvalues. Data suggest that $N_{q}(n, k)$ and $N_{q}(n, l), k \neq l$ do not have any eigenvalue in common. In other words, the Laplacian of $C_{q}(n)$ seems to have $\sum_{k=0}^{\lfloor n / 2\rfloor}(n-2 k+1)=$ $(\lfloor n / 2\rfloor+1)(\lceil n / 2\rceil+1)$ distinct eigenvalues (in contrast to the $q=1$ case, where the Laplacian has $n+1$ distinct eigenvalues), with each of the $n-2 k+1$ eigenvalues of $N_{q}(n, k)$ having multiplicity $\left[\begin{array}{c}n \\ k\end{array}\right]_{q}-\left[\begin{array}{c}n \\ k-1\end{array}\right]_{q}, k=0,1, \ldots,\lfloor n / 2\rfloor$. Is this true and can it be proved without explicitly writing down the eigenvalues?

\section{Positivity}

In this section we define certain combinatorial objects and a matchings type polynomial based on them with the property that an appropriate positive specialization satisfies the recurrence (5).

Consider the set $[n, \bar{n}]=\{1,2, \ldots, n\} \cup\{\overline{1}, \overline{2}, \ldots, \bar{n}\}$ of $2 n$ elements. We are going to recursively define a set $S(n)$ of certain subsets of $[n, \bar{n}]$. The cardinality of an element of $S(n)$ will be between 0 and $n$ (inclusive) and will have the same parity as $n$. Define $S(0)=\{\emptyset\}, S(1)=\{\{1\},\{\overline{1}\}\}$ and, for $n \geqslant 1$,

$$
S(n+1)=\{X \cup\{n+1\}: X \in S(n)\} \cup\{X \cup\{\overline{n+1}\}: X \in S(n), n \notin X\} \cup S(n-1) .
$$

It is easy to show by induction that $|S(n)|=2^{n}$ and that the number of elements of $S(n)$ not containing $n$ is $2^{n-1}$. Let $\mathbf{x}=\left(x_{1}, x_{2}, \ldots\right), \mathbf{y}=\left(y_{1}, y_{2}, \ldots\right)$, and $t$ be indeterminates. For $n \geqslant 0$ define the following polynomial

$$
P(n, \mathbf{x}, \mathbf{y}, t)=\sum_{X \in S(n)}\left(\prod_{i \in X \cap\{1, \ldots, n\}} x_{i}\right)\left(\prod_{\bar{i} \in X \cap\{\overline{1}, \ldots, \bar{n}\}} y_{i}\right) t^{\frac{n-|X|}{2}} .
$$

Example We have

$$
\begin{aligned}
& S(2)=\{\emptyset,\{1,2\},\{\overline{1}, 2\},\{\overline{1}, \overline{2}\}\} \\
& S(3)=\{\{1\},\{\overline{1}\},\{3\},\{\overline{3}\},\{1,2,3\},\{\overline{1}, 2,3\},\{\overline{1}, \overline{2}, 3\},\{\overline{1}, \overline{2}, \overline{3}\}\} .
\end{aligned}
$$


Thus $P(0, \mathbf{x}, \mathbf{y}, t)=1, P(1, \mathbf{x}, \mathbf{y}, t)=x_{1}+y_{1}, P(2, \mathbf{x}, \mathbf{y}, t)=t+\left(x_{1} x_{2}+y_{1} x_{2}+y_{1} y_{2}\right)$ and

$$
P(3, \mathbf{x}, \mathbf{y}, t)=\left(x_{1}+y_{1}+x_{3}+y_{3}\right) t+\left(x_{1} x_{2} x_{3}+y_{1} x_{2} x_{3}+y_{1} y_{2} x_{3}+y_{1} y_{2} y_{3}\right)
$$

The recursive structure of $S(n)$ yields the following recurrence for the polynomials $P$.

Theorem 4.1 We have

$$
P(n+1, \mathbf{x}, \mathbf{y}, t)=\left(x_{n+1}+y_{n+1}\right) P(n, \mathbf{x}, \mathbf{y}, t)-\left(x_{n} y_{n+1}-t\right) P(n-1, \mathbf{x}, \mathbf{y}, t), \quad n \geqslant 1 .
$$

Proof Write the right hand side as $T(1)+T(2)+T(3)$, where

$$
\begin{aligned}
& T(1)=x_{n+1} P(n, \mathbf{x}, \mathbf{y}, t), \\
& T(2)=y_{n+1} P(n, \mathbf{x}, \mathbf{y}, t)-x_{n} y_{n+1} P(n-1, \mathbf{x}, \mathbf{y}, t), \\
& T(3)=t P(n-1, \mathbf{x}, \mathbf{y}, t) .
\end{aligned}
$$

Let $X \in S(n+1)$. In the expansion of the left hand side $P(n+1, \mathbf{x}, \mathbf{y}, t)$, consider the term corresponding to $X$ :

$$
\left(\prod_{i \in X \cap\{1, \ldots, n+1\}} x_{i}\right)\left(\prod_{\bar{i} \in X \cap\{\overline{1}, \ldots, n+1\}} y_{i}\right) t^{\frac{n+1-|X|}{2}} .
$$

We consider three cases:

(i) $n+1 \in X$ : the term above will appear exactly once in $T(1)$ and not at all in $T(2)$ and $T(3)$.

(ii) $\overline{n+1} \in X$ : the term above will appear exactly once $T(2)$ and not at all in $T(1)$ and $T(3)$.

(iii) $X \in S(n-1)$ : The term above will appear exactly once in $T(3)$ and not at all in $T(1)$ and $T(2)$.

It is clear that there are no other terms corresponding to $X$ on the right hand side. The result follows.

Given $n, k \in \mathbb{N}$ with $k \leqslant n / 2$ define

$$
\begin{aligned}
& d_{q}(n, k)=\left([n-k]_{q},[n-k-1]_{q}, \ldots,[k]_{q}, 0,0, \ldots\right), \\
& e_{q}(n, k)=\left([k]_{q},[k+1]_{q}, \ldots,[n-k]_{q}, 0,0, \ldots\right) .
\end{aligned}
$$

We now prove Theorem 1.2.

Theorem 4.2 Let $n, k \in \mathbb{N}$ with $k \leqslant n / 2$ and let $k \leqslant j \leqslant n-k+1$. Then

$$
F_{q}(n, k, j)=P\left(n-k-j+1, d_{q}(n, k), e_{q}(n, k),[k]_{q}[n-k+1]_{q}\right),
$$

showing that the coefficients of $F_{q}(n, k, j)$ are nonnegative. 
Proof Denote the right hand side of (11) by $E_{q}(n, k, j)$. We verify that $E_{q}(n, k, j)$ satisfies the same recurrence (5) (and the same initial conditions $(3),(4))$ as $F_{q}(n, k, j)$. It follows from the formulas for $P(0, \mathbf{x}, \mathbf{y}, t)$ and $P(1, \mathbf{x}, \mathbf{y}, t)$ in the example above that $(3),(4)$ are satisfied.

Now note the following alternate expression for the square of the singular values:

$$
\begin{aligned}
q^{k}[j+ & 1-k]_{q}[n-k-j]_{q} \\
& =\left([j+1]_{q}-[k]_{q}\right)\left([n-j]_{q}-q^{n-k-j}[k]_{q}\right) \\
& =[j+1]_{q}[n-j]_{q}-[k]_{q}\left([n-j]_{q}+q^{n-k-j}[j+1]_{q}-q^{n-k-j}[k]_{q}\right) \\
& =[j+1]_{q}[n-j]_{q}-[k]_{q}[n-k+1]_{q} .
\end{aligned}
$$

We now have from Theorem 4.1 and (12) that, for $k \leqslant j<n-k, E_{q}(n, k, j)$ is

$$
\begin{aligned}
& =\left([j]_{q}+[n-j]_{q}\right) E_{q}(n, k, j+1)-\left([j+1]_{q}[n-j]_{q}-[k]_{q}[n-k+1]_{q}\right) E_{q}(n, k, j+2) \\
& =\left([j]_{q}+[n-j]_{q}\right) E_{q}(n, k, j+1)-\left(q^{k}[j+1-k]_{q}[n-k-j]_{q}\right) E_{q}(n, k, j+2) .
\end{aligned}
$$

Thus $E_{q}(n, k, j)$ satisfies $(5)$. The result follows.

Example Let $n=3$ and $k=0$. Then

$$
d_{q}(3,0)=\left([3]_{q},[2]_{q},[1]_{q},[0]_{q}, 0,0, \ldots\right), \quad e_{q}(3,0)=\left([0]_{q},[1]_{q},[2]_{q},[3]_{q}, 0,0, \ldots\right) .
$$

Thus $F_{q}(3,0,1)=P\left(3, d_{q}(3,0), e_{q}(3,0), 0\right)=[3]_{q}[2]_{q}[1]_{q}$.

Now let $n=3$ and $k=1$. Then

$$
d_{q}(3,1)=\left([2]_{q},[1]_{q}, 0,0, \ldots\right), \quad e_{q}(3,1)=\left([1]_{q},[2]_{q}, 0,0, \ldots\right) .
$$

Thus $F_{q}(3,1,1)=P\left(2, d_{q}(3,1), e_{q}(3,1),[1]_{q}[3]_{q}\right)=[1]_{q}[3]_{q}+[2]_{q}[1]_{q}+[1]_{q}[1]_{q}+[1]_{q}[2]_{q}=$ $4+3 q+q^{2}$.

We now discuss the connection between the $q=1$ case of Theorem 1.1 and formula (2), yielding an eigenvalue interpretation to (2). Substituting $q=1$ in Theorem 2.1 we get an orthogonal SJB $J(1, n)$ of $V(B(n))$ satisfying (9) with $q=1$. For $i=0,1, \ldots, n$, define the following vectors in $V(B(n))$ :

$$
\begin{aligned}
u_{i} & =\sum_{X \in B(n)_{i}} X, \\
v_{i} & =\frac{u_{i}}{\left\|u_{i}\right\|} .
\end{aligned}
$$

Let $R(n)$ be the subspace $V(B(n))$ spanned by $\left\{v_{0}, \ldots, v_{n}\right\}$. Clearly, $\operatorname{dim}(R(n))=n+1$. Elements of $R(n)$ are called radial vectors in $V(B(n))$ (this notion is defined, in the context of lumpable Markov chains, in [CST] (see Sections 1.10 and 2.6)). It is easy to see that $R(n)$ is invariant under the adjacency and Laplacian operators $A$ and $L$ of $C(n)$. 
In [CST] it is shown that the eigenvalues of $A: R(n) \rightarrow R(n)$ are $n-2 i, i=0,1, \ldots, n$. Since $C(n)$ is regular of degree $n$ it follows that the eigenvalues of $L: R(n) \rightarrow R(n)$ are $2 i, i=0,1, \ldots, n$.

Now note the following:

(i) The unique SJB in $J(1, n)$ starting at rank 0 is $\left(u_{0}, \ldots, u_{n}\right)$ and thus the matrix of $L: R(n) \rightarrow R(n)$ with respect to $\left\{v_{0}, \ldots, v_{n}\right\}$ is $N_{1}(n, 0)$.

(ii) We have $N_{1}(n, k)=2 k I+N_{1}(n-2 k, 0)$. This can be easily seen by checking that the matrix entries on both sides of the identity are equal. The rows and columns of the matrices on the two sides of the identity are indexed by different sets (of the same cardinality) but the intended meaning is clear.

It follows that the eigenvalues of $N_{1}(n, k)$ are $2 k, 2(k+1), \ldots, 2(n-k)$ and thus we have $F_{1}(n, k, k)=\prod_{j=k}^{n-k}(2 j), 0 \leqslant k \leqslant n / 2$.

Substituting $q=1$ in Theorem 4.2 we now get the following identity

$$
P\left(n-2 k+1, d_{1}(n, k), e_{1}(n, k), k(n-k+1)\right)=2^{n-2 k+1} \cdot k \cdot(k+1) \cdots(n-k) .
$$

Finally, we consider the Grassmann graphs (see section 12.4.3 in Brouwer and Haemers $[\mathbf{B H}])$. Let $0 \leqslant m \leqslant n / 2$. The Grassmann graph $C_{q}(n, m)$ is defined to be the graph with $B_{q}(n)_{m}$ as the vertex set and with two vertices $X, Y \in B_{q}(n)_{m}$ connected by an edge iff $\operatorname{dim}(X \cap Y)=m-1$. The adjacency eigenvalues and hence the Laplacian eigenvalues, since $C_{q}(n, m)$ is regular, were determined by Delsarte $[\mathbf{D}]$. We now show that using identity (12) the Laplacian eigenvalues can be written down in an elegant form yielding a positive formula for the (rooted) complexity of $C_{q}(n, m)$. For the sake of completeness we do not assume Delsarte's [D] result but give a proof using Theorem 2.1.

Theorem 4.3 Let $0 \leqslant m \leqslant n / 2$. The Laplacian eigenvalues of $C_{q}(n, m)$ are given by

$$
[k]_{q}[n-k+1]_{q}, k=0, \ldots, m,
$$

with respective multiplicities $\left[\begin{array}{c}n \\ k\end{array}\right]_{q}-\left[\begin{array}{c}n \\ k-1\end{array}\right]_{q}$. Thus

$$
\bar{c}\left(C_{q}(n, m)\right)=\prod_{k=1}^{m}\left([k]_{q}[n-k+1]_{q}\right)^{\left[\begin{array}{l}
n \\
k
\end{array}\right]_{q}-\left[\begin{array}{c}
n \\
k-1
\end{array}\right]_{q}} .
$$

Proof Let $U, D$ denote the up, down operators on $V\left(B_{q}(n)\right)$ and let $A, L: V\left(B_{q}(n)_{m}\right) \rightarrow$ $V\left(B_{q}(n)_{m}\right)$ denote the adjacency and Laplacian operators of $C_{q}(n, m)$. Let $I$ denote the identity operator on $V\left(B_{q}(n)_{m}\right)$. Then it is easy to see that $V\left(B_{q}(n)_{m}\right)$ is invariant under $D U$ and we have the identity

$$
A=D U-[n-m]_{q} I .
$$


Let $J(q, n)$ be an orthogonal SJB of $V\left(B_{q}(n)\right)$ satisfying the conditions of Theorem 2.1. For $k=0,1, \ldots, m$ define

$$
\begin{gathered}
J(q, n, m, k)=\{v \in J(q, n): r(v)=m \text { and the Jordan chain containing } v \\
\text { starts at rank } k\} .
\end{gathered}
$$

Let $W(q, n, m, k)$ be the subspace spanned by $J(q, n, m, k)$. Then we have an orthogonal direct sum decomposition

$$
V\left(B_{q}(n)_{m}\right)=\oplus_{k=0}^{m} W(q, n, m, k) .
$$

Clearly $\operatorname{dim}(W(q, n, m, k))=\left[\begin{array}{c}n \\ k\end{array}\right]_{q}-\left[\begin{array}{c}n \\ k-1\end{array}\right]_{q}$. We have from (13) and Theorem 2.1

$$
A(v)=\left(q^{k}[m+1-k]_{q}[n-k-m]_{q}-[n-m]_{q}\right) v, v \in W(q, n, m, k) .
$$

Now the degree of a vertex of $C_{q}(n, m)$ is $q[m]_{q}[n-m]_{q}$ (see Lemma 3 in [D]) and thus, for $v \in W(q, n, m, k)$, we have $L(v)=\theta v$, where

$$
\begin{aligned}
\theta & =q[m]_{q}[n-m]_{q}-q^{k}[m+1-k]_{q}[n-k-m]_{q}+[n-m]_{q} \\
& =[n-m]_{q}\left(1+q[m]_{q}\right)-q^{k}[m+1-k]_{q}[n-k-m]_{q} \\
& =[n-m]_{q}[m+1]_{q}-q^{k}[m+1-k]_{q}[n-k-m]_{q} \\
& =[k]_{q}[n+1-k]_{q}
\end{aligned}
$$

where in the last step we have used identity (12). The result now follows on observing that $[k]_{q}[n-k+1]_{q} \neq[l]_{q}[n-l+1]_{q}$ for $k \neq l$.

\section{Acknowledgement}

I thank Professors Alexander Schrijver, Navin Singhi, and Thomas Zaslavsky for their comments on an earlier version of this paper. I am grateful to Sivaramakrishnan Sivasubramanian for several useful discussions about the positivity of the polynomials $F_{q}(n, k, j)$.

\section{References}

[BH] A. E. Brouwer, and W. H. Haemers, Spectra of graphs, Springer, 2012.

[B] L. Butler, A unimodality result in the enumeration of subgroups of a finite abelian group, Proc. American Math. Soc., 101 (no. 4): 771-775 (1987).

[CST] T. Ceccherini-Silberstein, F. Scarabotti, and F. Tolli, Harmonic analysis on finite groups, Cambridge University Press, 2008.

[D] P. Delsarte, Association schemes and t-designs in regular semilattices, J. Combinatorial Theory, Series A, 20 (no. 2): 230-243 (1976). 
[E] K. Engel, Sperner theory, Cambridge University Press, 1997.

[JL] G. James, and M.Liebeck, Representations and Characters of Groups, Cambridge University Press, 2001.

[K] W. M. Kantor, On incidence matrices of finite projective and affine spaces, Math. Z., 124:315-318 (1972).

[P] R. A. Proctor, Representations of $\mathfrak{s l}(2, \mathbb{C})$ on posets and the Sperner property, SIAM J. Alg. Discr. Methods, 3:275-280 (1982).

[S] A. Schrijver, New code upper bounds from the Terwilliger algebra and semidefinite programming, IEEE Tran. Information Theory, 51: 2859-2866 (2005).

[St1] R. P. Stanley, Enumerative Combinatorics - Volume 1, Second Edition, Cambridge University Press, 2012.

[St2] R. P. Stanley, Enumerative Combinatorics - Volume 2, Cambridge University Press, 1999.

[T] P. Terwilliger, The incidence algebra of a uniform poset, in Coding theory and design theory, Part I, volume 20 of IMA Vol. Math. Appl., pages 193-212. Springer, New York, 1990. 